\title{
ESTUDIO RETROSPECTIVO DE CASOS DE INSUFICIENCIA CARDÍACA EN ADULTOS DEL 2011 AL 2015 EN UN HOSPITAL DOMINICANO
}

\section{Retrospective Study of Cases of Heart Failure in Adults from 2011 to 2015 in a Dominican Hospital}

\author{
Grisel Canahuate ${ }^{a}$, Marcelle Elías Purcell ${ }^{b}$, Carlos B. Ruiz-Matuk ${ }^{c}$
}

Recibido: 6 de junio, 2019 • Aprobado: 2 de septiembre, 2019

Cómo citar: Canahuate G, Elías Purcell M, Ruiz-Matuk CB. Estudio retrospectivo de casos de insuficiencia cardíaca en adultos del 2011 al 2015 en un hospital dominicano. cysa [Internet]. 16 de junio de 2020 [citado 17 de junio de 2020];4(2):45-54. Disponible en: https://revistas.intec.edu.do/index.php/cisa/article/view/1764

\section{Resumen}

Introducción: la insuficiencia cardíaca (IC) es un síndrome caracterizado por un funcionamiento inadecuado de las capacidades cardíacas, que se asocia con discapacidad. Sus múltiples etiologías y factores de riesgo varían geográficamente, por lo que se plantea conocer las características clínicas de la IC en la República Dominicana, con el objetivo de sugerir mejoras en los servicios de salud.

Métodos: se identificaron 2,119 casos de pacientes con IC en el Hospital General de la Plaza de la Salud (HGPS) y con base en esta población se calculó una muestra de 326 casos, a partir de un intervalo de confianza de $95 \%$ y un margen de error de $5 \%$. Partiendo del cálculo de la muestra, se seleccionaron aleatoriamente 378 casos. Este sobre muestreo se realizó previendo la posible eliminación de algunos casos en los análisis finales. Luego de esto, se procedió a la extracción de datos.

Resultados: el estudio mostró que la edad promedio fue de 69 años. Se evidenciaron diversos factores de riesgos predominantes como la hipertensión arterial $87.5 \%$, cardiopatía isquémica $50.4 \%$, valvulopatías $40 \%$, y la diabetes mellitus un $34.7 \%$. También se observaron conductas de riesgo en las que predominan el uso de tabaco (23.7\%), obesidad (17.4\%) y consumo de alcohol

a Unidad de Insuficiencia Cardíaca, Departamento de Cardiología, Hospital General de la Plaza de la Salud, Santo Domingo, República Dominicana. Correo-e: gcanahuate@hgps.org.do,

ORCID: 0000-0002-7750-8203

\begin{abstract}
Introduction: Heart failure (HF) is a syndrome characterized by inadequate functioning of the cardiac capacities associated with disability. Its multiple etiologies and risk factors vary geographically, so it is proposed to know the clinical characteristics of HF in the Dominican Republic with the aim of suggesting improvements in health services.
\end{abstract}

Methods: 2,119 cases of patients with HF were identified in Hospital General de la Plaza de la Salud (HGPS) and based on this population size, a sample of 326 cases was calculated, based on a $95 \%$ confidence interval and a $5 \%$ margin of error. Based on the sample calculation, 378 cases were randomly selected. This oversampling was carried out anticipating the possible extraction of some cases in the final analyzes. After sampling, data was obtained.

Results: The study showed that the average age was 69 years. Diverse risk factors were evident predominantly arterial hypertension $87.5 \%$, ischemic heart disease $50.4 \%$, valvulopathies $40 \%$, and diabetes mellitus $34.7 \%$. Risk behaviors were also observed in which the use of tobacco predominated $(23.7 \%)$, obesity $(17.4 \%)$ and alcohol

\footnotetext{
b Docente-Investigadora, Decanato de Investigación, Universidad Iberoamericana, Santo Domingo, República Dominicana

Correo-e: melias@clinicalresearchrd.com, ORCID: 0000-0002-8382-7681

${ }^{\mathrm{c}}$ Director, Decanato de Investigación, Universidad Iberoamericana, Santo Domingo, República Dominicana

Correo-e: c.ruiz4@unibe.edu.do, ORCID: 0000-0003-2681-4953
} 
(7.8\%). El síntoma más frecuente fue disnea con $84.7 \%$. La fracción de eyección promedio fue $48 \%$. El tratamiento más común fue los diuréticos. La mortalidad registrada fue $11.1 \%$.

Discusión: este estudio evidencia una alta ocurrencia de conductas y factores de riesgo, cuya intervención y subsecuente disminución pudiera significar cambios importantes a la prevalencia de la IC en el país.

Palabras clave: insuficiencia cardíaca; morbimortalidad; hipertensión arterial; factores de riesgo cardiovasculares.

\section{Introducción}

La insuficiencia cardíaca (IC) es un conjunto de afectaciones clínicas, debido a daños tanto estructurales como funcionales del sistema cardíaco, que resulta en disminución de la función de bomba y aumento de las presiones intracavitarias y que lleva a una inadecuada respuesta a la demanda del organismo ${ }^{1,2}$ Los signos y síntomas más comunes como el edema, la disnea y la fatiga, son debido a las respuestas tanto circulatorias como neurohormonales producto de la disfunción del sistema circulatorio. ${ }^{1}$ Las causas son muy variables y en ocasiones son el resultados de múltiples factores predisponentes o enfermedades comórbidas como la enfermedad coronaria, la hipertensión arterial, la diabetes, las valvulopatías, la toxicidad cardíaca e inclusive diferentes tipos de enfermedades infecto-contagiosas que conducen a un deterioro agudo o progresivo de la función cardíaca. ${ }^{1}$ Por sus condiciones clínica, la IC es capaz de incapacitar a los pacientes tanto en el ámbito laboral como en el hogar, limitando una buena calidad de vida tanto física como mentalmente. Por lo tanto, es considerada una problemática de salud a nivel mundial, con una prevalencia actual de más de 5,8 millones de pacientes en los EE.UU. y más de 23 millones a nivel mundial. ${ }^{3}$ Además, 2,4 millones de pacientes que son hospitalizados en Estados Unidos, tienen también un diagnóstico primario o secundario de IC, y cerca de 300,000 muertes anuales son debido a esta enfermedad. $^{3}$ consumption $(7.8 \%)$. The most frequent symptom was dyspnea with $84.7 \%$. The average ejection fraction was $48 \%$. The most frequent treatment was diuretics. The recorded mortality was $11.1 \%$.

Discussion: This study evidences a high occurrence of behaviors and risk factors whose intervention and subsequent decrease could mean important changes to the prevalence of HF in the country.

Keywords: Heart failure; morbimortality; arterial hypertension; cardiovascular risk factors.

De acuerdo con los datos del estudio PRICE, realizado en España, la prevalencia de la IC es alrededor de un 7-8 \% en personas mayores de 45 años. ${ }^{4}$ En los Estados Unidos, los adultos tiene una prevalencia de un 1-2\% de IC, pero en los ancianos, por encima de los 75 años, esta cifra puede alcanzar hasta un $10 \% .^{5}$ En la recopilación de varios estudios de IC realizados en América Latina y el Caribe se estimó de manera general una prevalencia de un $1,01 \%$, y una mortalidad hospitalaria de un 11,67 \% siendo la enfermedad de Chagas y la cardiopatía isquémica las principales causas, sin olvidar que existe una gran variabilidad en las condiciones geográficas, étnicas y económicas de estos países. ${ }^{5-7}$ Por lo tanto, como estos estudios provienen de un grupo limitado de países de la región, su generalización a otras poblaciones latinoamericanas como la República Dominicana, debe tomarse con precaución. ${ }^{5}$

En el 2001 se hizo una publicación en una revista dominicana sobre la incidencia de la IC en 4,877 pacientes tratados en el Departamento de Cardiología del Hospital Dr. Francisco E. Moscoso Puello durante los años 1997-1998, siendo la incidencia de $2.1 \%{ }^{8}$ En el país se desconoce la incidencia y prevalencia actual, así como hospitalizaciones y mortalidad por esta enfermedad. Por lo que es un reto la producción de estudios observacionales que puedan orientar sobre nuestras principales problemáticas sanitarias. Este estudio es una contribución, al describir las características de esta enfermedad y su priorización en los servicios de salud por su alta mortalidad, incapacidad laboral, altos costos 
hospitalarios y múltiples tratamientos. De igual manera, proporciona datos actualizados sobre la IC en el país, con miras a aportar al acervo científico nacional.

Es de resaltar que, a pesar de los avances en el tratamiento y la evolución a largo plazo de las diferentes enfermedades cardíacas, la morbimortalidad de la IC no ha descendido significativamente. ${ }^{4}$ Esto debido, probablemente, a que estos avances han aumentado la esperanza de vida de los pacientes, en consecuencia, existen más comorbilidades como la hipertensión arterial, enfermedad renal crónica y diabetes mellitus, entre otras. ${ }^{4}$ Estas comorbilidades van produciendo alteraciones crónicas, como son cambios en la remodelación del miocardio, cambios inflamatorios y obstructivos a nivel vascular, que, de no ser debidamente tratados, dificultan la mejoría de los pacientes con IC.

Podemos extrapolar los resultados de un estudio realizado en países latinoamericanos como el alto índice de pobreza; el alto costo de vida que conduce a cambios en el estilo de vida, entre ellos la ingesta de alimentos hipercalóricos y el sedentarismo; factores como bajos gastos de salud por habitante; y pocas medidas para el desarrollo de políticas preventivas de salud. ${ }^{6,7}$ Pero aun compartiendo los factores de riesgo de carácter socioeconómico, mencionados anteriormente, típicos de los países en desarrollo como el nuestro, debemos definir cuáles otros factores de riesgo más específicos están contribuyendo a que nuestros pacientes desarrollen IC. ${ }^{9-11}$ Esto es prioritario para poder dirigir los recursos hacia un mejor control de dichos factores. ${ }^{12}$ De esta forma ir trabajando en torno a estos, identificar la población más vulnerable y desarrollar intervenciones para mitigar o, hasta cierto punto, evitar algunos de sus efectos. ${ }^{13}$ De esta manera podemos analizar cada aspecto que conduzca al desarrollo de esta condición y así poder elaborar nuevos lineamientos que contribuyan a una disminución de la incidencia, prevalencia y gasto público anual de la insuficiencia cardíaca. ${ }^{14-15}$ Haciendo énfasis en guías actuales, enfocadas en desarrollar estrategias preventivas dirigidas a la modificación del comportamiento de la población relacionado a la insuficiencia cardíaca. ${ }^{16}$

El objetivo de este estudio fue conocer las características demográficas, factores de riesgo predisponentes y asociadas a la insuficiencia cardíaca y mortalidad por la enfermedad en un hospital céntrico de referencia en la República Dominicana. Se realiza este estudio para que los datos sirvan como base para crear lineamientos de mejora a nuestros servicios de salud en cuanto a esta enfermedad. De igual manera se buscó conocer cuál es el tratamiento utilizado en estos pacientes e identificar oportunidades de mejora de cara a características poblacionales.

\section{Método}

Se revisó el sistema de record médico electrónico del hospital y de 357,254 pacientes atendidos en el mismo, se identificaron los casos que cumplían con el diagnóstico de insuficiencia cardíaca entre los años 2011 y 2015, siendo la tasa de prevalencia de 593 por 100,000 pacientes. Se identificaron 2,119 casos que cumplían con los criterios de inclusión y con base en este tamaño poblacional se calculó una muestra de 326 casos, a partir de un intervalo de confianza de $95 \%$ y un margen de error de $5 \%$. Partiendo del cálculo de la muestra, se seleccionaron aleatoriamente 378 casos. Este sobre muestreo se realizó previendo la posible eliminación de algunos casos en los análisis finales, por razón de datos perdidos. Se obtuvo la información de los expedientes a partir de un sistema de base de datos electrónico (LOLCLI 9000) utilizando la determinación de los diagnósticos de insuficiencia cardíaca, insuficiencia cardíaca congestiva, miocardiopatía dilatada, y enfermedad cardíaca hipertensiva con insuficiencia cardíaca. Se evaluó por medio de la historia clínica y las evoluciones, cuáles fueron los motivos de la visita al hospital, cuáles características clínicas se determinaron para hacer el diagnóstico de insuficiencia cardíaca y qué estudios avalan este diagnóstico. 
Luego de corroborar el diagnóstico de insuficiencia cardíaca, se determinaron las características demográficas de estos pacientes, específicamente la edad, sexo y lugar de procedencia. Además se levantó la información de sus antecedentes personales patológicos, principalmente si eran hipertensos, diabéticos, o si padecían de insuficiencia renal, dislipidemia o infarto al miocardio, además de hábitos tóxicos, y si previo a su visita al hospital tenían algunos de los siguientes diagnósticos: insuficiencia cardíaca per se, cardiopatía isquémica dilatada, miocardiopatía dilatada idiopática, miocardiopatía periparto o post parto, miocardiopatía hipertrófica, cardiopatías congénitas, valvulopatías o miocarditis. Se determinó, además, el tratamiento previo que estos pacientes llevaban y el tratamiento con el que fueron manejados por última vez en el hospital.

El levantamiento de la información fue llevado a cabo por unos 20 asistentes de investigación, quienes cursaban la carrera de medicina de la Universidad Iberoamericana (UNIBE) luego de firmar un documento de confidencialidad y recibir entrenamiento sobre el estudio. Los datos fueron digitados en plantillas estandarizadas creadas en Excel. Luego de los procesos de control de calidad (limpieza de códigos posibles y limpieza de contingencia), las plantillas fueron exportadas a SPSS para analizar los datos.

El estudio obtuvo la aprobación del Comité de Ética de UNIBE y del Hospital General de la Plaza de la Salud (HGPS) (acta no. 42). De igual manera, se obtuvo la aprobación del Consejo Nacional de Bioética (CONABIOS) (registro no. 046-2016) de la República Dominicana. Los datos fueron digitados protegiendo la identidad de cada paciente, trabajando por medio de números asignados a cada record. Estos datos tuvieron una doble revisión del autor, para garantizar la confiabilidad de la información de cada participante. De igual manera, se utilizaron los datos para la realización de análisis inferenciales que pueden servir como guía para entender mejor las relaciones entre variables.

\section{Resultados}

Para los análisis finales se utilizaron datos de 378 pacientes, quienes tienen una edad promedio de 69.1 años, lo que indica la gran mayoría de nuestros pacientes con insuficiencia cardíaca son envejecientes. En cuanto a las características sociodemográficas $48.7 \%$ de casos de IC eran hombres y el $51.3 \%$ mujeres. Por otro lado, al observar la procedencia se puede notar que la mayoría de los pacientes eran de la ciudad en el $64.8 \%$ de los casos, mientras que el $34.1 \%$ acudía al hospital desde las diferentes provincias del país. Además, hubo un $0.8 \%$ de casos en los cuales los pacientes provenían de otros países (tabla 1 ).

Tabla 1. Características sociodemográficas

\begin{tabular}{|l|l|l|}
\hline \multicolumn{1}{|c|}{ Características sociodemográficas } & No. & $\%$ \\
\hline EDAD & & \\
\hline Promedio & 69.1 & N/A \\
\hline SEXO & & \\
\hline Femenino & 194 & $51.3 \%$ \\
\hline Masculino & 184 & $48.7 \%$ \\
\hline TOTAL & 378 & $100 \%$ \\
\hline PROCEDENCIA & & \\
\hline Ciudad & 245 & $64.8 \%$ \\
\hline Provincia & 129 & $34.1 \%$ \\
\hline Otros países & 3 & $0.8 \%$ \\
\hline Se desconoce & 1 & $0.3 \%$ \\
\hline TOTAL & 378 & $100 \%$ \\
\hline
\end{tabular}

Fuente: elaboración propia.

Pudimos observar que la mayoría (71.9 \%) de los pacientes ya conocían su diagnóstico y eran tratados para esta enfermedad, mientras que el $28.1 \%$ lo desconocía. En las características modificables pudimos observar que el tabaquismo juega un rol importante, ya que representa un $23.7 \%$ de los casos, seguido de la obesidad con un $17.4 \%$, el alcoholismo con un $7.8 \%$ y un $0.8 \%$ reportaba consumo de drogas. Se observó también que un $2.8 \%$ de pacientes recibía quimioterapia, y un $1.7 \%$ radioterapia (figura 1). 


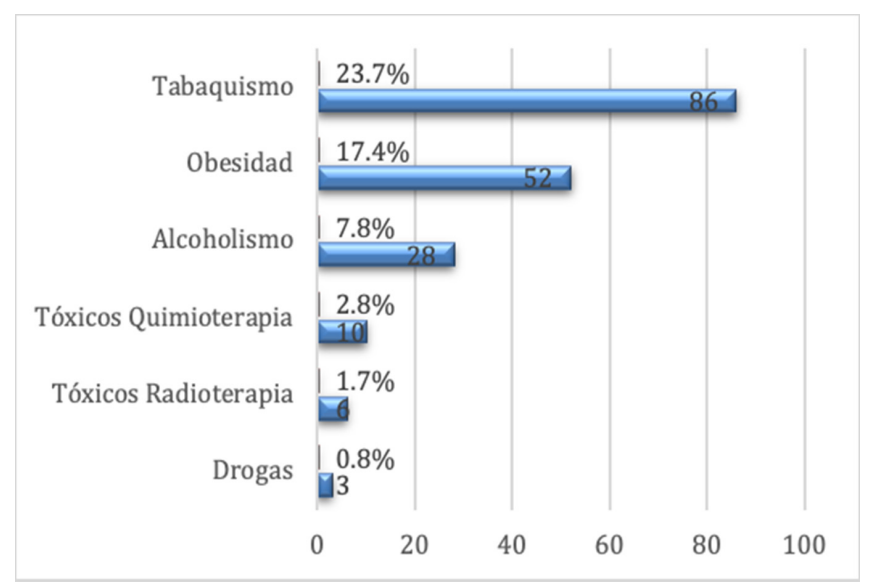

Figura 1. Características Modificables

Fuente: elaboración propia.

Se realizó un análisis exhaustivo de los antecedentes personales de los pacientes, la hipertensión arterial es el factor de riesgo primordial en esta enfermedad con un $87.5 \%$ de los pacientes reportando esta comorbilidad. Sin embargo, la cardiopatía hipertensiva, aunque en un alto porcentaje, solo se observó en un $59.2 \%$, lo que probablemente quiere decir que la hipertensión arterial es un factor de riesgo independiente del daño estructural que pueda producirse a nivel del músculo cardíaco. Muy de cerca le sigue la cardiopatía isquémica con un $50.4 \%$, donde además notamos que el 67 $\%$ de estos estaban revascularizados, $6.7 \%$ con derivación aorto coronarias y hasta un $59.9 \%$ con angioplastia con colocación de stents y un 33.5 $\%$ no fueron revascularizados, sin poder definir la causa. Las valvulopatías ocupaban un $40 \%$, y de estas la valvulopatía mitral es la más frecuente con $37 \%$, seguida de la valvulopatía aórtica con $38 \%$, la tricúspides afectada en un $27 \%$, y la pulmonar en un $4 \%$. Además, pudimos establecer la prevalencia de cardiopatía reumática encontrada en esta población que fue de un $4 \%$ (figura 2).

La diabetes mellitus se observó en un $34.7 \%$, las arritmias cardíacas un $32.4 \%$, la anemia en un 30 $\%$ sin ninguna especificación, la insuficiencia renal crónica en un $28.6 \%$, la miocardiopatía dilatada idiopática $24.3 \%$, las hiperlipidemias $22 \%$, la enfermedad pulmonar obstructiva crónica $15.4 \%$, el cáncer un $8 \%$, el tromboembolismo pulmonar un $4.2 \%$, la endocarditis $2.5 \%$, las cardiopatías congénitas $2.1 \%$ y en menor grado la miocardiopatía peri o post parto en un $1 \%$, la miocardiopatía hipertrófica en $0.5 \%$, y la miocardiopatía por tóxicos $0.3 \%$ (figura 2 ).

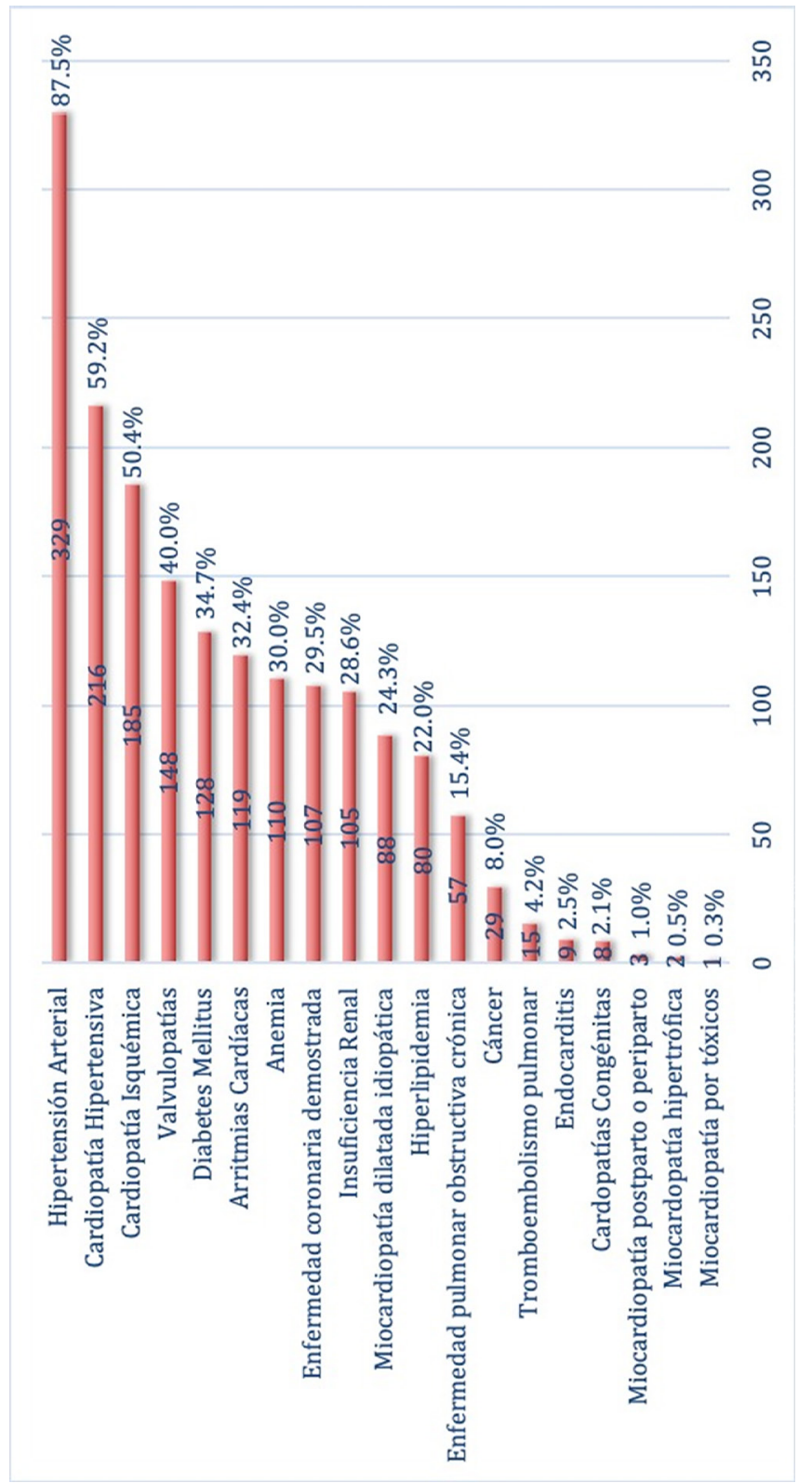

Figura 2. Factores de riesgo observados

Fuente: elaboración propia. 
De las cardiopatías congénitas se destacan la comunicación interauricular con $28.6 \%$, la aorta bicúspide y la comunicación interventricular ambas con $14.3 \%$, en menor grado se observaron la persistencia del conducto arterioso, el prolapso mitral, y el canal auriculoventricular, cada una con $0.3 \%$. Entre las arritmias tenemos que la más común es la fibrilación auricular con un $47 \%$, seguido por las arritmias ventriculares en un $32 \%$ y las supraventriculares (excluyendo la fibrilación auricular) en un $21 \%$ (figura 2).

En el análisis de los métodos diagnósticos se registra que la disnea es el síntoma principal reportado con un $84.7 \%$ de ocurrencia, seguido del edema con un $69.5 \%$, y los estertores crepitantes en un $61.2 \%$, estando en última instancia la disnea paroxística nocturna con un $14.7 \%$ y el ápex desplazado en un $9 \%$. En la radiografía de tórax era común la cardiomegalia con $62.1 \%$, y la congestión pulmonar en un $30.8 \%$ (figura 3 ).

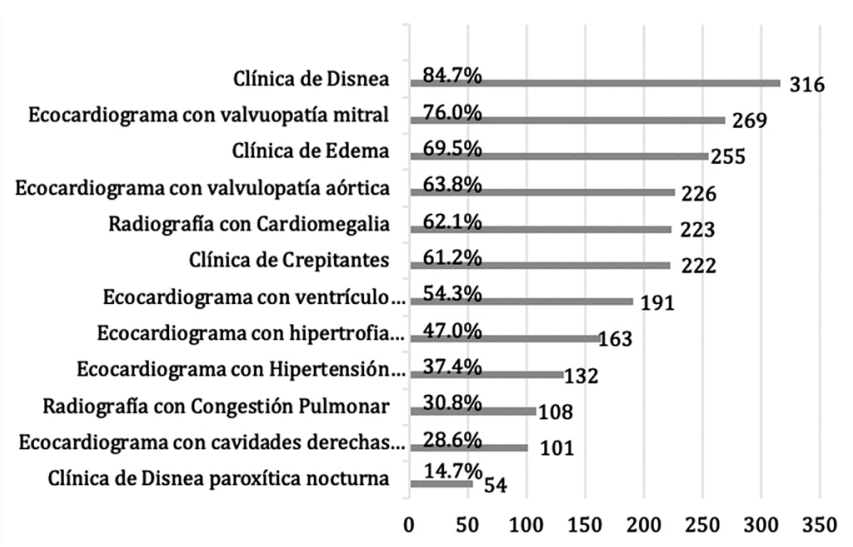

Figura 3. Métodos utilizados para confirmar o hacer el diagnóstico

Fuente: elaboración propia.

El ecocardiograma evidenció que la fracción de eyección promedio fue de $48 \%$, lo que indica que la mayoría de los pacientes solo tenían ligera disfunción sistólica. Además, en el ecocardiograma observamos que el ventrículo izquierdo estaba dilatado en el $54.3 \%$ de los casos, y la dilatación de cavidades derechas se evidenció en un $28.6 \%$ de los casos. Había hipertrofia en el $47 \%$ de los pacientes e hipertensión pulmonar en el $37.4 \%$ (figura 3).

En los laboratorios obtuvimos valores promedios ligeramente elevados como glicemia en $123 \mathrm{mg} / \mathrm{dl}$, creatinina de $1.46 \mathrm{mg} / \mathrm{dl}$, colesterol total de 182 $\mathrm{mg} / \mathrm{dl}$, colesterol HDL de $42 \mathrm{mg} / \mathrm{dl}$, colesterol LDL de $115 \mathrm{mg} / \mathrm{dl}$, y triglicéridos en $138 \mathrm{mg} / \mathrm{dl}$. Los valores de PRO-BNP sí estuvieron elevados obteniéndose un valor promedio de 13,663 , tomando en cuenta que este solo se realizó en un porcentaje muy bajo a pesar de su conocida relación con el pronóstico de los pacientes con fallo cardíaco. ${ }^{17}$

En el análisis del tratamiento inicial, los fármacos más utilizados fueron los diuréticos, que se prescribieron a un $85.6 \%$ de los casos estudiados. De los cuales la furosemida fue la más común con $63.3 \%$, seguido de hidroclorotiazida en un $12.2 \%$, y la bumetanida con un $7.8 \%$.

En segundo lugar, se encontró el uso de antiagregantes plaquetarios en un $64.3 \%$. El más utilizado fue el clopidogrel en un $59.3 \%$, y la aspirina quedó en un segundo plano con $38.7 \%$.

Los betabloqueadores se utilizaron en un $47.1 \%$ de los casos, dentro de los cuales el de mayor uso fue el carvedilol en un $47.8 \%$, seguido del bisoprolol en un $31.5 \%$, atenolol en un $12.4 \%$. Los bloqueadores de los receptores de angiotensina se utilizaron en un $37.3 \%$, de los cuales el más frecuente fue el losartán con $50.4 \%$, seguido de candesartán con un $10.5 \%$, el irbesartán $5.2 \%$.

Similar a los anteriores, los inhibidores de la enzima convertidora de angiotensina se utilizaron en el $35.2 \%$ de los casos predominando enalapril en un $30.1 \%$, seguido de captopril en $26.3 \%$, el ramipril en $24.8 \%$ y lisinopril en $18.0 \%$. 
Otros medicamentos también utilizados fueron la digoxina en un $34.9 \%$, la espironolactona en un $17.2 \%$. Además, los anticoagulantes orales como la warfarina en $9.7 \%$ y el rivaroxaban en un $3.1 \%$. Cabe señalar medicamentos cuyo uso fue de $0 \%$ como la ivabradina, a pesar de su conocida disminución de las hospitalizaciones y mortalidad. ${ }^{18}$

Se recopilaron otros tratamientos por su relación con las comorbilidades como las estatinas, que se utilizaron en un $28.3 \%$, siendo la más frecuente la rosuvastatina con un $61.7 \%$ de los mismos, seguido de la simvastatina con un $32.7 \%$.

Otros tipos de terapéuticas fueron la colocación de desfibrilador en $4.2 \%$ de los casos, la colocación de un resincronizador en $4.5 \%$, la ablación de las arritmias en $1.1 \%$, el tratamiento del cáncer con radioterapia en $0.8 \%$, y con quimioterapia en $3.1 \%$.

\section{Análisis de tratamiento final}

En el análisis del tratamiento final, los más utilizados fueron los diuréticos $(99.2 \%)$, principalmente la furosemida con un $63.3 \%$. Dentro de los antiagregantes plaquetarios (con un $79.3 \%$ de uso), los más utilizados son el clopidogrel con un $60.1 \%$, y la aspirina, con $36.5 \%$. Los betabloqueadores se utilizaron en un $53.4 \%$ de los casos, siendo el de mayor uso el carvedilol en un $63.3 \%$, seguido del bisoprolol en un $37.1 \%$.

Los bloqueadores de los receptores de angiotensina se utilizaron en un $41.3 \%$, siendo, dentro de los bloqueadores el losartán el más frecuente, con $45.5 \%$, seguido de candesartán con un $24.4 \%$. Los inhibidores de la enzima convertidora de angiotensina se utilizaron en el $47.4 \%$.

Otros medicamentos también utilizados fueron la digoxina en un $37.6 \%$, la espironolactona en un $22 \%$. Los anticoagulantes aumentaron considerablemente su uso, el rivaroxaban a un $12.9 \%$ y la warfarina a $64.9 \%$. Se recopilaron otros tratamientos como las estatinas que se utilizaron en un $33.6 \%$ siendo la más frecuente la rosuvastatina con un $23.4 \%$. Cabe señalar que medicamentos cuyo uso fue de $0 \%$, inicialmente, como la ivabradina aumentó a un $3.4 \%$ su utilidad al final.

Otros tipos de terapéuticas fueron la colocación de desfibrilador en un $5.3 \%$, la colocación de un resincronizador en $5 \%$, la ablación de las arritmias en $1.1 \%$ y, finalmente, el trasplante cardíaco con $0.3 \%$.

La mortalidad fue de un $11.1 \%$ predominando las causas cardíacas con $10.3 \%$ y las no cardíacas solo con un $0.8 \%$. El número de hospitalizaciones en promedio fue de $2.21(D E=2.41)$, con un mínimo de cero a un máximo de 5 hospitalizaciones. El promedio de días hospitalizados fue de 4.42 días $(D E=4.21)$. Es importante destacar que la mortalidad pudo haber sido superior a la cifra expresada, ya que el paciente pudo haber fallecido en otra institución de salud. El país no cuenta con un récord único que permita el registro universal de pacientes y así obtener información de mejor calidad para fines de seguimiento clínico e investigación.

Con respecto a la mortalidad, se realizó un conjunto de pruebas de independencia chi cuadrado para ver si existía una asociación entre esta variable y los valores de Pro-BNP, de creatina y la fracción de eyección, encontrándose solo significación estadística en la relación entre mortalidad y valor de creatinina $(=4.145, p=.042)$. Un conjunto de análisis análogos se realizó para detectar asociación entre el uso de tratamientos o medicamentos en función de la procedencia del paciente (rural o urbano). Solo se encontró asociación entre el uso de nitratos y la procedencia del paciente $(=5.667, p=.017)$. 


\section{Discusión}

La insuficiencia cardíaca se ha convertido mundialmente en una verdadera problemática de salud que necesita atención por la carga económica que representa para la sociedad. La elaboración de estudios sobre el tema contribuye a la búsqueda de tratamientos puntuales que logren la mejoría de los pacientes y así obtener una mejor calidad de vida, disminuir las hospitalizaciones, mortalidad e incluso ser reintegrados a la sociedad.

Lo que podemos aplicar de la IC y sus directrices viene dado por estudios realizados en países post industrializados como Estados Unidos y países de Europa occidental; en mucho menos proporción en otros países latinoamericanos, la mayoría en vías de desarrollo con altos niveles de desigualdad. La República Dominicana no había jugado un rol sustancial en estas investigaciones, por lo tanto, carecíamos de estadísticas que identificaran las principales causas nacionales que desempeñan un papel en esta enfermedad. ¿Cómo vamos a poder tratarla adecuadamente cuando las guías que tenemos están basadas en poblaciones étnicamente muy diferentes a las nuestras, y desconocemos si estos lineamientos son realmente aplicables a nuestra población caribeña?

El estudio arrojó que dentro de las características demográficas no hubo predominancia de sexo de manera significativa, y la edad promedio estuvo por encima de los 65 años lo que quiere decir es más frecuente en la población envejeciente como en otros países desarrollados. Dentro de las características clínicas principales se destacaron sobre todo la hipertensión arterial, la cardiopatía hipertensiva, la cardiopatía isquémica, las valvulopatías y la diabetes mellitus, y en moderada proporción están las arritmias, el tabaquismo, y la insuficiencia renal.
Algo muy importante que se observó fue que se realiza el diagnóstico utilizando la clínica, así como procedimientos especializados de baja complejidad. Entre las características clínicas que se toman en consideración están el edema, la disnea y crepitantes. También con laboratorios simples habituales, y con un Pro-BNP, además de una radiografía de tórax, electrocardiograma y ecocardiograma se pueden hacer los diagnósticos diferenciales principales para determinar la causa de la IC. Los medicamentos más utilizados siguen siendo los diuréticos, a estos le siguen betabloqueadores, los inhibidores de la enzima convertidora de angiotensina y los bloqueadores de los receptores de angiotensina, así como la digoxina y antiagregantes plaquetarios. Esto implica que la comunidad médica sigue los lineamientos de las guías internacionales, aunque permanece aún un alto porcentaje de subutilización. ${ }^{19}$ Sin embargo, medicamentos de última generación que han demostrado disminuir la mortalidad y hospitalizaciones en estos pacientes como la ivabradina, están siendo poco utilizados. ${ }^{19}$ Esta subutilización puede deberse a que los mismos no son siempre asequibles a la población y tampoco tienen cobertura para los planes de salud local básicos. Otros tratamientos como terapias de ablación de arritmias, desfibriladores y resincronizadores, así como el trasplante cardíaco están en un porcentaje bajo. Estas alternativas terapéuticas tienen disponibilidad limitada, así como altos costos en el país. Estas características socioeconómicas más la alta prevalencia de IC en poblaciones de adultos mayores con múltiples comorbilidades crean escenarios de mayor complejidad para el logro de objetivos terapéuticos óptimos. El análisis de la mortalidad y alta tasa nos ayuda a definir la prioridad que debería tener esta enfermedad y cuáles son las posibles causas de esta alta tasa. Nuestros recursos deben estar centrados en el control de los factores de riesgo y conductuales asociados con la enfermedad y la accesibilidad a nivel nacional de los mejores tratamientos disponibles. 


\section{Conclusiones}

Creemos urgente continuar con la elaboración de estudios prospectivos a gran escala con nuestra población, identificando las modalidades terapéuticas farmacológicas y conductuales que permiten evidenciar mejoras significativas. De esta manera, se podrán crear nuevas estrategias para la prevención y tratamiento de la insuficiencia cardíaca y formular políticas sobre cuáles enfermedades debemos priorizar en los servicios de salud por su alta mortalidad, incapacidad laboral y altos costos en general, incluyendo los intrahospitalarios. Un ejemplo sería crear programas especiales en conjunto con salud pública, donde se puedan subsidiar medicamentos de buena calidad, tipo antihipertensivos, hipoglucemiantes y de enfermedades crónicas, para que sean más asequibles a la población con menos recursos.

\section{Bibliografía}

1. Ceia F, Fonseca C, Mota T, et al. Prevalence of chronic heart failure in Southwestern Europe: The EPICA study. Eur J Heart Fail. 2002;4(4): 531-9.

2. European Society of Cardiology. Guidelines for the diagnosis and treatment of acute and chronic heart failure. Eur Heart J. 2016;37(27):2129200. Doi:10.1093/eurheartj/ehw128

3. Bui AL, Horwich TB, Foranow GC. Epidemiology and risk profile of heart Failure. Nat Rev Cardiol. 2011;8(1):30-41. Doi: 10.1038/ nrcardio.2010.165

4. Sánchez MA, Crespo MG, Galvan E, et al. Prevalencia de la insuficiencia cardiaca en la población general española mayor de 45 años. Estudio PRICE. Rev Esp cardiol. 2008;61(10): 1041-9. Doi: 10.1157/13126044
5. Ciapponi A, Alcaraz A, Calderón M, et al. Carga de enfermedad de la insuficiencia cardiaca en América Latina: revisión sistemática y metanálisis. Rev Esp Cardiol. 2016;69(11):1051-60. Doi: 10.1016/j.recesp.2016.04.045

6. Bocchi EA, Arias A, Verdejo H, et al. Interamerican Society of Cardiology. The reality of heart failure in Latin America. J Am Coll Cardiol. 2013;62(11):949-58. Doi: 10.1016/j. jacc.2013.06.013

7. Reddy KS, Yusuf S. Emerging Epidemic of Cardiovascular Disease in Developing Countries. Circulation. 1998;97:596-601. Available from: https://doi.org/10.1161/01.CIR.97.6.596

8. Romero-Estrella A. Incidencia de Insuficiencia Cardiaca en la Consulta de cardiología del Hospital Dr Francisco Moscoso Puello. Rev med dom. 2001;62(1):38-40.

9. Banegas JR, Rodríguez-Artalejo F, Guallar-Castillón P. Situación epidemiológica de la insuficiencia cardiaca en España. Rev Esp Cardiol Supl. 2006;6(C):4-9. Doi: 10.1157/13092028

10. Rodríguez GC, Barrios V, Aznar J, et al. Características clínicas de los pacientes diagnosticados de insuficiencia cardiaca crónica asistidos en atención primaria. Estudio CARDIOPRES. Rev Clin Esp. 2007;207(7):337-40. Doi: 10.1016/ S0014-2565(07)73402-1

11. Sayago I, García F, Segovia J. Epidemiología de la insuficiencia cardiaca en España en los últimos 20 años. Rev Esp Cardiol. 2013;66(8):649-56. Doi: 10.1016/j.recesp.2013.03.014

12. Recio J, Alegre J, Fernández T. Estudio prospectivo de los enfermos ingresados por insuficiencia cardíaca en una sala de hospitalización de medicina interna. Rev Clin Esp. 2004;204(7):362-4. 
13. Llorens P, Martín F, González J, et al. Perfil clínico del paciente con insuficiencia cardiaca aguda atendido en los servicios de urgencias: Datos preliminares del Estudio EAHFE. Emergencias 2008;20:154-63.

14. Blecker S, Paul M, Taksler G, et al. Heart Failure Associated Hospitalizations in the United States. J. Am. Coll. Cardiol. 2013;61(12):125967. Doi: $10.1016 /$ j.jacc.2012.12.038

15. Ambrosy AP, Fonarow GC, Butler J, et al. The global health and economic burden of hospitalizations for heart failure: lessons learned from hospitalized heart failure registries. J Am Coll Cardiol. 2014;63(12):1123-33. Doi: 10.1016/j. jacc.2013.11.053

16. Sayago I, García F, Segovia J. Epidemiología de la insuficiencia cardiaca en España en los últimos 20 años. Rev Esp Cardiol. 2013;66(8):649-56. Doi: 10.1016/j.recesp.2013.03.014
17. Oremus M, Don-Wauchope A, McKelvie R, et al. BNP and NT-proBNP as prognostic markers in persons with chronic stable heart failure. Heart Fail Rev. 2014;19(4):471-505. Doi: 10.1007/ s10741-014-9439-6

18. Swedberg K, Komajda M, Bohm M, et al. Ivabradine and outcomes in chronic heart failure (SHIFT). Lancet. 2010;376(9744):875-85. Doi: 10.1016/S0140-6736(10)61198-1

19. American College of Cardiology and American Heart Association. Guideline for the Management of Heart Failure. Circulation. 2013;128: e240-e327. Available from: https://doi.org/10. 1161/CIR.0b013e31829e8776

* El financiamiento para este estudio fue proporcionado por Novartis. Ninguno de los autores tiene conflicto de intereses.

** Los investigadores desean agradecer a los voluntarios y equipos administrativos de ambas instituciones por su apoyo en la realización de este proyecto. 